\title{
Eigenspace Method by Autoassociative Networks for Object Recognition
}

\author{
Takamasa Yokoi, Wataru Ohyama, \\ Tetsushi Wakabayashi, and Fumitaka Kimura \\ Faculty of Engineering, Mie University 1515 Kamihama, Tsu 514-8507, Japan \\ yokoi@hi.info.mie-u.ac.jp
}

\begin{abstract}
This paper studies on a new eignespace method which employs autoassociative networks for object recognition. Five layered autoassociative network is available to obtain a manifold on the minimum square error hypersurface which approximates a distribution of learning sample. Recognition experiments were performed to show that the manifold of rotating object is obtained by learning and the objects, such as a mouse and a stapler, are correctly recognized by the autoassociative networks. It is also shown that the accuracy of approximating closed manifold and the accuracy of recognition are improved by emploing multiple autoassociative networks each of which is trained by a partition of the learning sample.The property and the advantage of the five layered autoassociative network are demonstrated by a comparative study with the nearest neighbor method and the eigenspace method.
\end{abstract}

\section{Introduction}

The object recognition techniques play essential roles in widely raging applications from inspection and classification of industrial parts to vision system for autonomous mobile robot.

One of the typical object recognition techniques is the nearest neighbor method which compares an input image with multiple template images of each object captured a priori for variety of positions and illuminating condition. A drawback of the nearest neighbor method is that the required computation time and storage increases with the number of template images which increases rapidly depending on the degree of freedom of the variations.

To solve the problem Murase et al.[1] proposed a parametric eigenspace method which compresses an input image onto the eigenspace. The method parametrically represents a sequence of continuously varying images as a manifold in the eigenspace spanned by a small number of pricipal components of the variation to reduce the computation time and storage. However, since the eigenspace is derived by the K-L transformation, which is linear, the dimensionality of the eignespace tends to be higher than the intrinsic dimensionality of the variation. While the parametric representation is suitable for variations related to explicit parameters such as rotation, it is impossible to represent nonparametric or hidden parametric variations. 
This paper studies on a new eignespace method which employs autoassociative networks for object recognition. The autoassociative network is an artificial neural network having the same number of neurons in input and output layers, and the less in the middle (hidden) layers. The network is trained using the input vector itself as the desired output by the back propagation method [2]. This training leads to organize a dimension reduction network between the input layer and the middle layer, and a restoration network between the middle layer and the output layer (Fig.1). The autoassociative neural network was applied to image compression and dimension reduction [3-5]. The five layered autoassociative network performs dimension reduction by nonlinear mapping and the input pattern is mapped onto a manifold on minimum square error hypersurface which approximates the distribution of the learning sample. The square error between the input and the output of the network stands for squared distance between the input and the manifold, by which the minimum distance classification is easily performed for the input object [6].

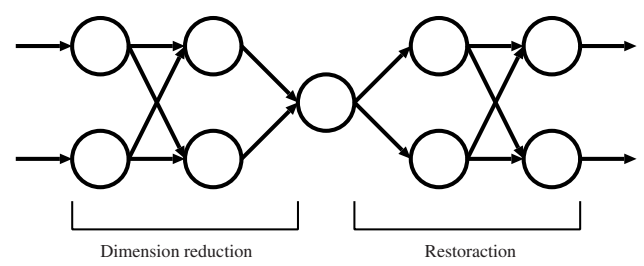

Fig. 1. Autoassociative neural network

Recognition experiments were performed to show that the manifold of rotating object is obtained by learning and the objects, such as a mouse and a stapler, are correctly recognized by the autoassociative networks. It is also shown that the accuracy of approximating closed manifold and the accuracy of recognition are improved by emploing multiple autoassociative networks each of which is trained by a partition of the learning sample. The property and the advantage of the five layered autoassociative network are demonstrated by a comparative study with the nearest neighbor method and the eigenspace method.

Section 2 outlines the learning and the classification by means of the autoassociative network, and Sect.3 describes the learning of the manifold yielded by a rotating object. Section 4 describes the experiments and the results of the object recognition, and Sect.5 summarizes and concludes this work.

\section{Autoassociative Neural Networks}

The proposed method organizes a set of networks each of which are trained independently for each class using the feature vector of the class. As a result, the squared error between an input and the output is generally minimized by the network of the class to which the input pattern belongs. This property enables 
us to classify an unknown input pattern: The unknown pattern is fed to all networks, and is classified to the class with minimum squared error.

In contrast with the pattern recognition using the mutual associative networks, each autoassociative network is organized independently for each class, and the training load of the networks can be distributed to handle large scale pattern recognition problems. The first and the second layers of the five layered networks perform nonlinear encoding operation, and the fourth and the fifth layers the nonlinear decoding operation. The five layered networks studied in this paper have the same number of neurons in their second and fourth layers, and thus symmetric encoding and decoding networks.

Figure 2 shows an example of learning process of the five layered autoassociative networks for nonlinearly distributed two dimensional patterns. The axes of graphs represent the feature value $\left(x_{1}, y_{1}\right)$. The number of neurons in the input layer (first layer) to the output layer (fifth layer) is $2,2,1,2,2$ respectively. The input signal is samples of two dimensional feature vector from each class, and the same signal is given as the teaching signal (desired output), and the learning process is repeated independently for each class. Two curved line segments in this figure represent the trace of the output which are obtained by sweeping the output of hidden layers from 0 to 1 . Squares on the line segments represent the projections of the samples. These line segments converge to the medial axes of the distributions, which play the same role as the principal axes of the principal component analysis.

Figure 3 shows how to classify an input pattern by the converged autoassociative networks. An input pattern $\left(x_{1}, x_{2}\right)$ is given to the network of each

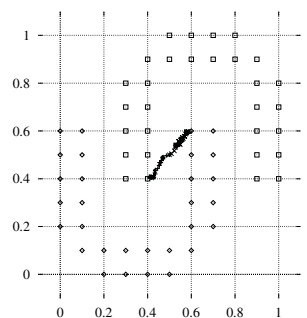

(a)Iteration: 1

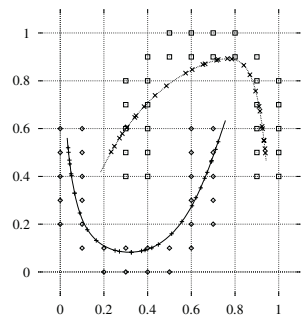

(c)Iteration: 13000

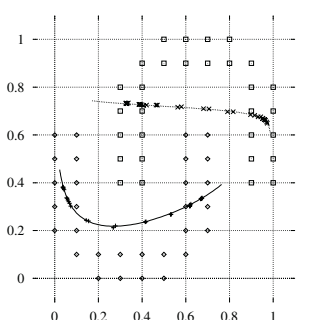

(b) Iteration: 6500

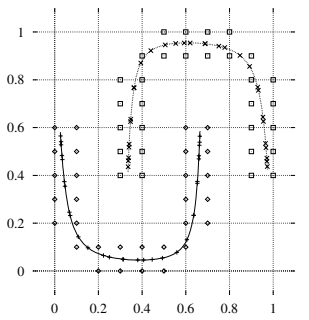

(d)Iteration: 4000000

Fig. 2. Learning process of five layered autoassociative neural networks 
class. The output $\left(y_{1}, y_{2}\right)$ and $\left(z_{1}, z_{2}\right)$ are the coordinates of $Y$, and $Z$ on the projection line respectively, and the squared distance are given by $\|Y-X\|^{2}$, and $\|Z-X\|^{2}$ respectively. The input pattern $X$ is classified to the class with the minimum distance, i.e. the class which minimizes the square error of the input and the output of the network, e.g. the left lower class in this figure. The output $u$ and $v$ from the hidden layers take the value from 0 to 1 .
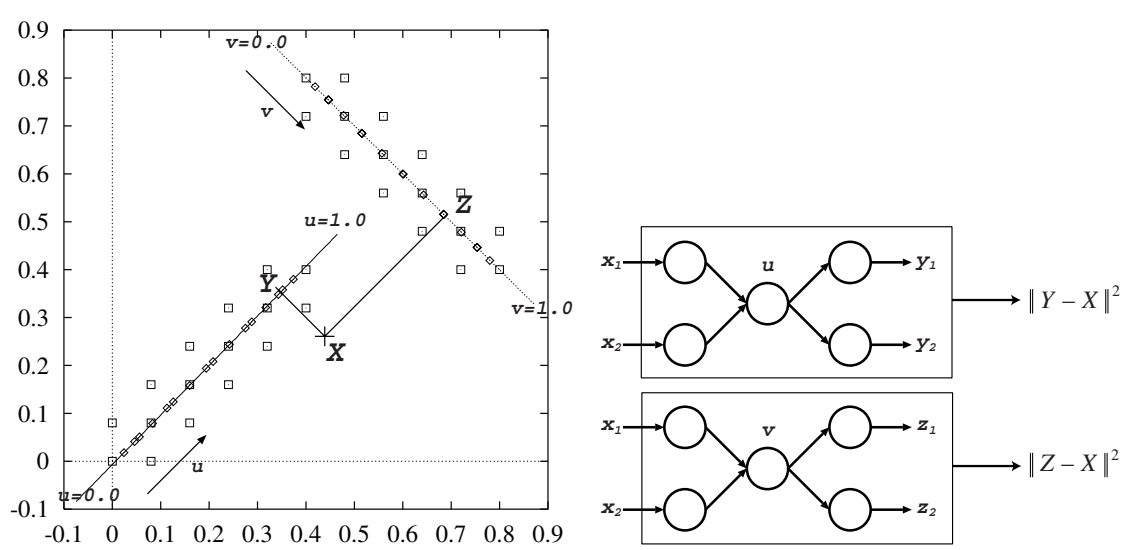

Fig. 3. Discrimination of unknown pattern by three layered autoassociative neural networks

Figure 4 shows example of dimension reduction and classification by five layered autoassociative networks for nonlinearly distributed patterns. This figure shows that the samples are projected to curved hypersurfaces (curved lines in two dimensional case) by five layered networks. An input pattern is classified to the class with the minimum squared error, i.e. the class with the nearest curves. While the nonlinearly distributed pattern can not be completely separated by the three layered networks, all samples can be separated by the five layered networks which can perform nonlinear projections (Fig. 4).

\section{Learning of the Eigenspace by the Autoassociative Network}

This section describes how the five layered autoassociative network learns the image sequence of an object continuously changing its appearence.

The learning sample of each object consists of 36 gray scale images of size $400 \times 400$ which are captured at every 10 degree of rotation on vertical axis. We used a computer controlled turn table to acquire the learning sample. Figure 5 shows the example of the learning sample. The input of the autoassociaive network is 100 dimensional feature vector composed of the average brightness in $10 \times 10$ blocks of the image. The feature vector is input to the autoassociaive 

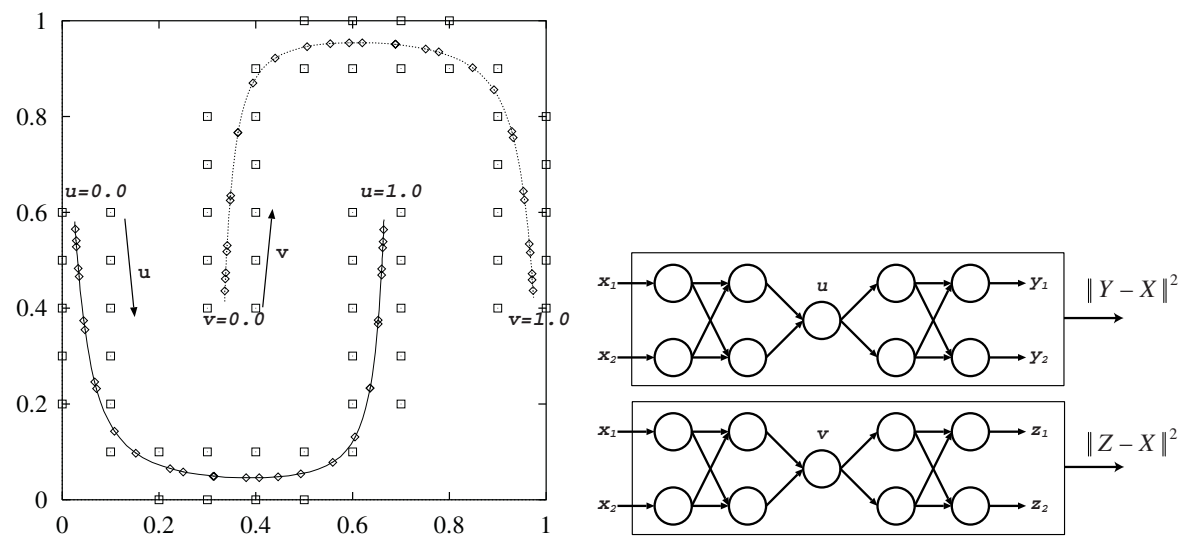

Fig. 4. Dimension reduction and discrimination by five layered autoassociative neural networks

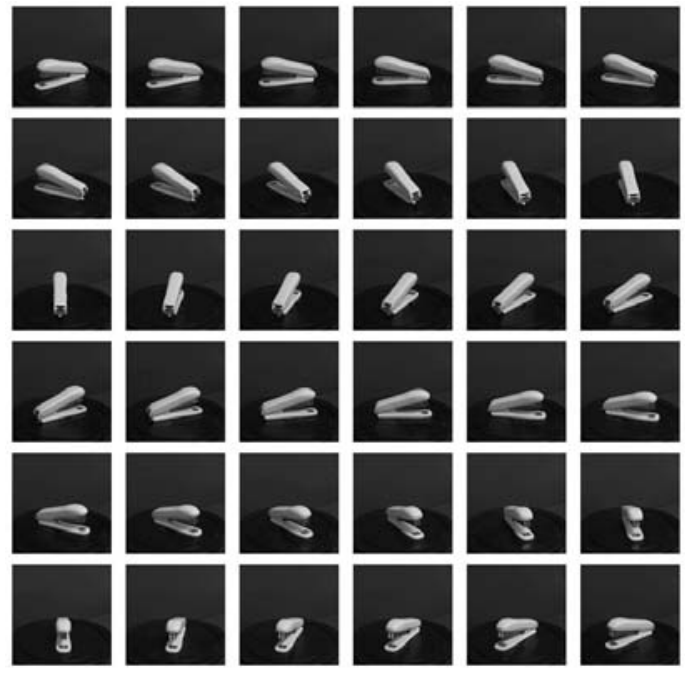

Fig. 5. Example of learning sample of a rotating object

network as an input signal and a desired output. The number of neurons of 5 layered network is 100,4,1,4,100 respectively. The network of this organization approximates the distribution of the learning sample in the feature space by four pieces of one dimensional curves.

Figures 5 and 6 show an example of rotating object and the distribution of the feature vectors. Figure 6 shows the distribution projected and visualized in the three demensional eigenspace obtained by the principal component analysis of the 100 dimensional feature distribution. The $x, y$, and $z$ axis respectivly stands for the principal components with largest three variances (eignvalues) of 


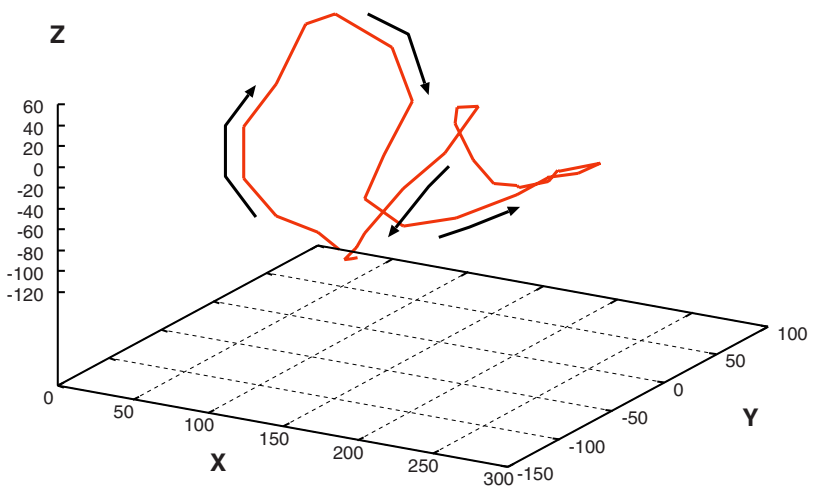

Fig. 6. Distribution of the learning sample in the eigenspace

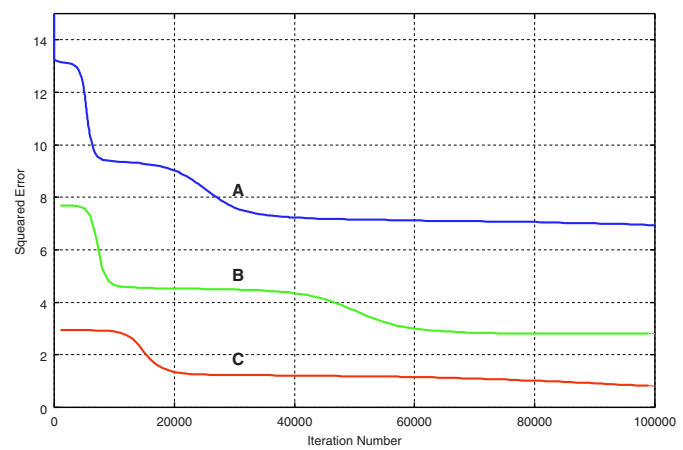

Fig. 7. The relationship between the squared error and the times of learning

the distribution. The feature vector of the rotating object draws a trajectory in the feature space. In order to impove the accuracy and the efficiency of approximating the closed manifold, the learning sample is divided into several groups and fed to separate networks.

Figure 7 shows the relationship between the number of learning and sum of the squeared error. The curve A, B and C in the figure shows the squared error when the learning sample is divided into 1,2 and 4 groupes, respectively. This figure shows that the more the number of groupes is the less the squared error is.

Figure 8 shows the distribution of the learning sample and the approximating curves obtained by the autoassociative networks. Figure $8(\mathrm{a}),(\mathrm{b})$ and (c) is the result for the case where the learning sample is devided into 1, 2 and 4 groupes, respectively. Each figure shows the three dimensional eignspace represented in the same way as in Fig. 6 and its projection to $\mathrm{x}-\mathrm{y}, \mathrm{y}-\mathrm{z}$ and $\mathrm{z}-\mathrm{x}$ planes for visual clarity's sake. The dotted line in the figure is the manifold on which the learning sample is distributed, and the solid line is the approximating curves of the manifold which is learned by the autoassociative networks. Each approximating 

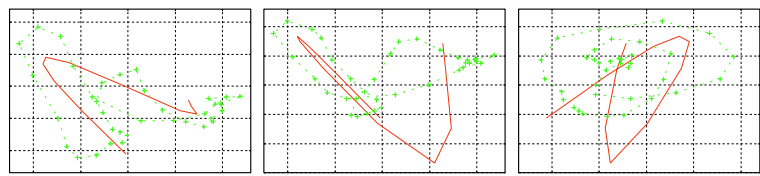

(a)Number of partition:1

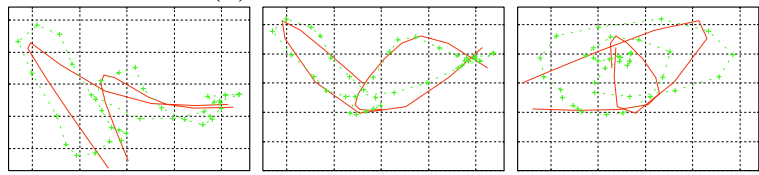

(b)Number of partition:2

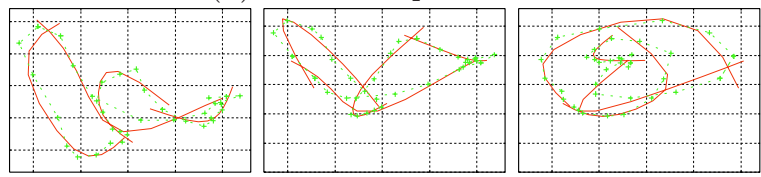

(c) Number of partition:4

Fig. 8. The approximating curves obtained by the autoassociative networks

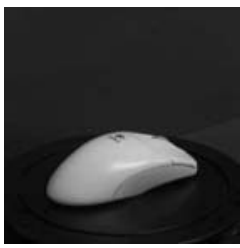

(a)Mouse

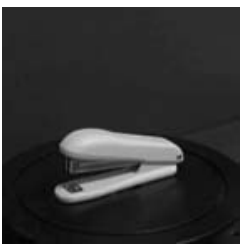

(b)Stapler

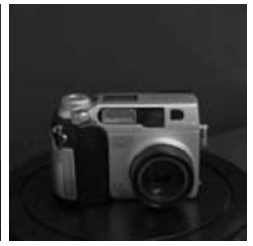

(c)Camera

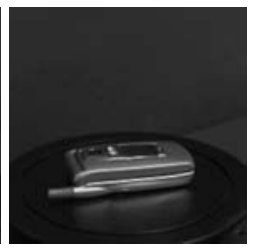

(d)Mobilephone

Fig. 9. Four classes of objects used in the recognition experiment

curve is the output of the network when the output of the middle hidden unit is swept from 0 to 1 as described in Sect.2. This figure shows that the more the number of groupes the more accurately the distribution is approximated.

\section{Object Recognition by the Autoassociative Networks}

To demonstrate the feasibility of the object recognition by the autoassociative networks, recognition experiment of four classes of objects shown in Fig.9 was performed.

Each sample for learning and test consists of 36 gray scale images per class, and were acquired in the same way as described in Sect.3. The learning sample and the test sample is different in the rotating angle by 5 degrees.

Recognition experiments were performed under the following five conditions:

1. Five layered autoassociative network (single segment)

2. Five layered autoassociative network (two segments)

3. Five layered autoassociative network (four segments)

4. Nearest neighbor method

5. Nearest neighbor method in eigenspace 
Table 1. The recognition rate of each method

\begin{tabular}{c|c}
\hline Method & Recognition rate(\%) \\
\hline Five layered autoassociative network (single segment) & 97.92 \\
Five layered autoassociative network (two segments) & 98.69 \\
Five layered autoassociative network (four segment) & 100.00 \\
Nearest neighbor method & 100.00 \\
Nearest neighbor method in eigenspace & 100.00 \\
\hline
\end{tabular}

Table 2. The result of recognition experiment with smaller learning samples

\begin{tabular}{c||c|c|c}
\hline \multirow{2}{*}{ Method } & \multicolumn{3}{c}{ Number of learning samples } \\
\cline { 2 - 4 } & 36 & 12 & 4 \\
\hline Five layered autoassociative networks(four segments) & $100.00 \%$ & $100.00 \%$ & $100.00 \%$ \\
Nearest neighbor method & $100.00 \%$ & $100.00 \%$ & $93.06 \%$ \\
Nearest neghbor method in eigenspace & $100.00 \%$ & $99.31 \%$ & $94.44 \%$ \\
\hline
\end{tabular}

Where "single segment" is a synonym of division into one group, and so on. Table 1 shows the recognition rate of each method.

mmThe five layered autoassociative networks with more segments achieved higher recognition rate. This result is justified by the fact that the closed manifold is more accurately approximated when separate networks are trained by divided learning samples. The nearest neghbor methods achieved $100 \%$ recognition rate both in original feature sapce and eigenspace.

Table 2 shows the result of recognition experiment when smaller learning samples were used.

The autoassociative networks keep high recognition rates for smaller sample case while the nearest neghbou methods deteriorate the performance.

\section{Concluding Remarks}

This paper studied on a new eignespace method which employs autoassociative networks for object recognition. The results of recognition experiments for four classes of objects demonstrated that:

1. the five layered autoassociative networks achieve higher recognition rate when separate networks are trained by divided learning samples because the closed manifold is more accurately approximated, and

2. they outperform the nearest neighbor methods in original space and the eigenspace with smaller learning samples.

Remaining future research topics are shown as follows,

1. Learning and recognition of object images which change with higher degree of freedom,

2. recognition of more classes of objects, and

3. pose estimation of the object 


\section{References}

1. Murase,H.,Nayar,S.:Visual Learning and Recognition of 3D Objects from Appearance.International Journal of Computer Vision,14-1(1995)5-24

2. Hassoun,M.:Fundamentals of Artificial Neural Networks.MIT Press,(1995)

3. Cottrell,G.,Munro,P.,Zipser,D.:Image compression by back-propagation: An example of extensional programing. Models of Cognition: A Review of Cognitive Science, 1(1989)208-240

4. Cottrell,G.,Munro,P.,Zipser,D.:Learning internal representations from gray-scale images:An example of extensional programing. Ninth Annual Conference of the Cognitive Science Society,(1987)462-473

5. DeMers,D.,Cottell,G.:Non-linear dimensionality reduction. Advances in Neural Information Processing Systems 5,(1992) 550-587

6. Kimura,F.,Inoue,S.,Wakabayashi,T.,Tsuruoka,S.,Miyake,Y.:Handwritten Numeral Recognition using Autoassociative Neural Networks. Proc. 14th International Conference on Pattern Recognition,1(1998)166-171 